\title{
Stability and Control of Relative Equilibria of Three-Spacecraft Magnetically Tethered Systems
}

\author{
I. I. Hussein* and A. M. Bloch ${ }^{\dagger}$
}

\begin{abstract}
In this paper we derive the reduced dynamical equations of motion for a planar threespacecraft magnetic formation. We derive necessary and sufficient conditions for the relative equilibria for the system. We then show that three spinning configurations satisfy these conditions. Each on of these equilibria describe a family of relative equilibria. Due to the complexity of the resulting equations, we also study the stability and controllability for a given choice of system parameters about one of the found equilibria. For the chosen system parameters, it is shown that the system is unstable yet controllable given a set of control inputs (magnetic dipole moments and control external torques). Finally, we derive linearization-based control laws to stabilize the system about the chosen equilibrium and for the chosen system parameters. This work sets the stage for a future general stability, controllability and control study of three-spacecraft magnetically tethered systems.
\end{abstract}

\section{Introduction}

Virtual-tether satellite constellations have received much interest in recent years. Virtual tethers can be created using distance action forces such as those created by electrostatic forces (see for example Ref. [1-5] and references therein) or magnetic potentials (see for example Ref. [6,7] and references therein). Virtual tethers require less power requirements for basic satellite maneuvers, have longer lifetimes, and avoid the use of physical tethers, which could be problematic for complex constellation shape changes.

This work builds on that by the authors in Ref. [8] for two-spacecraft magnetic systems. In this paper, we will study a three-spacecraft magnetic system, where each spacecraft is equipped with a magnetic coil that can be independently controlled using internal torques. The derivation of the basic equations of motion will first be performed. Conservation laws are investigated and conserved momentum variables (in the unactuated case) will be derived. Any such conservation laws will be used to reduce the size of the differential equations using Routh reduction. Conditions for the existence of relative equilibria (i.e., fixed configuration with constant spin rates) will be derived and solved to obtain all possible families of relative equilibria. As is always the case with magnetic systems, one expects these equilibria to be unstable. Due to the complexity of the general stability analysis, we will show through linearization and for a given choice of system parameters that the linearized equations are unstable for one of the derived equilibria, and, hence, that the nonlinear system itself is unstable. With this done, we will restrict ourselves to showing controllability of the shape dynamics of the system via magnetic and torque actuation. Finally, linearization-based control techniques are employed to stabilize the system about a chosen equilibrium and for a given choice of system parameters.

\section{The Three-Spacecraft Planar Problem}

\section{A. System Inertia Properties and Coordinates}

In this paper we consider a three-craft system. Each spacecraft is equipped with a superconducting coil that generates a magnetic field around the craft. The three magnetic fields interact to generate inter-spacecraft force interactions. These forces will be used to control the relative distances and orientations between the craft. Usually, the coil is such that it is free to rotate inside the spacecraft, separate from the spacecraft's

*Assistant Professor, Worcester Polytechnic Institute, Mechanical Engineering Department, 100 Institute Road, Worcester, MA 01609-2280. E-mail: ihussein@wpi.edu

$\dagger$ Alexander Ziwet Collegiate Professor of Mathematics; Chair, Dept.of Mathematics, University of Michigan, Ann Arbor, MI 48109. E-mail: abloch@umich.edu 
frame. Internal motors are used to rotate the superconducting coils relative to the spacecraft frame, which will have to respond to the coil motion due to conservation of angular momentum. For the sake of simplicty, we will ignore the spacecraft frame and assume that the coils are fixed to the frame. In place of internal motors, we will assume that the torques applied to the coils are external in nature. That is, the torques acting on the entire spacecraft using control moment gyroscopes or linear thruster pairs.

Hence, consider the three spacecraft planar system shown in Figure 1. The three craft are assumed identical with identical magnetic coils. Let $\mathbf{r}_{1}, \mathbf{r}_{2}, \mathbf{r}_{3}$ be the positions of the centers of mass of the craft. Let $\mathbf{q}_{\mathrm{cm}}$ be the position of the center of mass of the system:

$$
\mathbf{q}_{\mathrm{cm}}=\frac{M \mathbf{r}_{1}+M \mathbf{r}_{2}+M \mathbf{r}_{3}}{3 M}=\frac{1}{3}\left(\mathbf{r}_{1}+\mathbf{r}_{2}+\mathbf{r}_{3}\right),
$$

where $M$ is the total mass of each spacecraft. Let the relative position from spacecraft 1 to spacecraft 2 be given by

$$
\mathbf{q}_{1}=\mathbf{r}_{2}-\mathbf{r}_{1}
$$

and the relative position from spacecraft 1 to spacecraft 3 be given by

$$
\mathbf{q}_{2}=\mathbf{r}_{3}-\mathbf{r}_{1}
$$

In terms of $\mathbf{q}_{\mathrm{cm}}, \mathbf{q}_{1}$ and $\mathbf{q}_{2}$, the position vectors $\mathbf{r}_{1}, \mathbf{r}_{2}$ and $\mathbf{r}_{3}$ are given by

$$
\begin{aligned}
& \mathbf{r}_{1}=\mathbf{q}_{\mathrm{cm}}-\frac{\mathbf{q}_{1}}{3}-\frac{\mathbf{q}_{2}}{3} \\
& \mathbf{r}_{2}=\mathbf{q}_{\mathrm{cm}}+\frac{2 \mathbf{q}_{1}}{3}-\frac{\mathbf{q}_{2}}{3} \\
& \mathbf{r}_{2}=\mathbf{q}_{\mathrm{cm}}-\frac{\mathbf{q}_{1}}{3}+\frac{2 \mathbf{q}_{2}}{3},
\end{aligned}
$$

which give the velocities

$$
\begin{aligned}
& \dot{\mathbf{r}}_{1}=\dot{\mathbf{q}}_{\mathrm{cm}}-\frac{\dot{\mathbf{q}}_{1}}{3}-\frac{\dot{\mathbf{q}}_{2}}{3} \\
& \dot{\mathbf{r}}_{2}=\dot{\mathbf{q}}_{\mathrm{cm}}+\frac{2 \dot{\mathbf{q}}_{1}}{3}-\frac{\dot{\mathbf{q}}_{2}}{3} \\
& \dot{\mathbf{r}}_{2}=\dot{\mathbf{q}}_{\mathrm{cm}}-\frac{\dot{\mathbf{q}}_{1}}{3}+\frac{2 \dot{\mathbf{q}}_{2}}{3} .
\end{aligned}
$$

We will parameterize $\mathbf{q}_{\mathrm{cm}}$ by the center of mass cartesian coordinates $\mathbf{q}_{\mathrm{cm}}=(x, y)$. The relative position coordinate $\mathbf{q}_{1}, \mathbf{q}_{2}$ will be parameterized by the relative distance $\left(q_{1}, q_{2}\right)$ and the relative attitude angles $\left(\psi_{1}, \psi_{2}\right)$ as shown in Figure 1 . Also, as shown in the figure, the coils have angular positions of $\theta_{1}, \theta_{2}$ and $\theta_{3}$ relative to $\mathbf{q}_{1}$ and $\mathbf{q}_{2}$ as shown in the figure. The coils are assumed to have the same moments of inertia of $J$ each.

\section{B. Reduction of the Center of mass Motion}

We will assume that no linear forces act on the system other than those that produce pure torques. Hence, by conservation of linear momentum, the system's linear momentum is conserved. If the system is such that the center of mass is initially at rest, it will thus remain so for all time. We will assume that the system center of mass is such that $(x, y)=(0,0)$. This eliminates two coordinate variables in the ensuing analysis, which leaves us with 7 variables: $\left(q_{1}, q_{2}, q_{3}, q_{4}, q_{5}, q_{6}, q_{7}\right):=\left(q_{1}, q_{2}, \psi_{1}, \psi_{2}-\psi_{1}, \theta_{1}, \theta_{2}, \theta_{3}\right)$, where we note that instead of $\psi_{1}$ and $\psi_{2}$ we use the angles $q_{3}=\psi_{1}$ and $q_{4}=\psi_{2}-\psi_{1}$. The coordinates $q_{1}, q_{2}, q_{4}, q_{5}, q_{6}, q_{7}$ define the geometric shape of the system, while $q_{3}$ defines the over system orientation with respect to an inertial frame. 


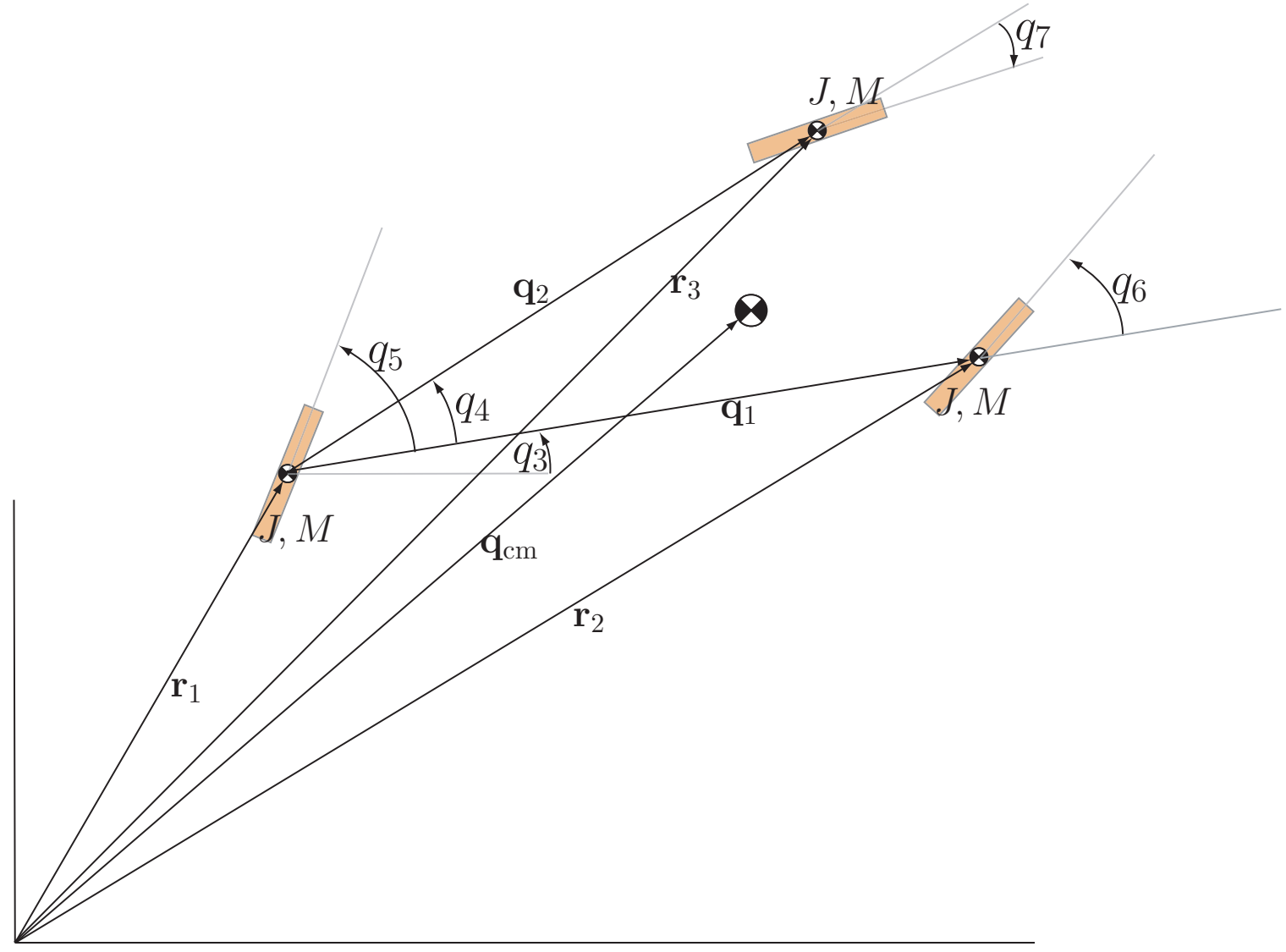

Figure 1. The three-spacecraft planar system.

\section{Kinetic Energy}

With the center of mass fixed at the origin, the kinetic energy for the two craft magnetically tethered system is given by

$$
\begin{aligned}
K\left(\dot{\mathbf{r}}_{1}, \dot{\mathbf{r}}_{2}, \dot{\theta}_{1}, \dot{\theta}_{2}, \dot{\theta}_{3}\right)= & \frac{M}{2}\left(\left\|\dot{\mathbf{r}}_{1}\right\|^{2}+\left\|\dot{\mathbf{r}}_{2}\right\|^{2}+\left\|\dot{\mathbf{r}}_{3}\right\|^{2}\right) \\
& +\frac{J}{2}\left(\left(\dot{q}_{3}+\dot{q}_{5}\right)^{2}+\left(\dot{q}_{3}+\dot{q}_{6}\right)^{2}+\left(\dot{q}_{3}+\dot{q}_{4}+\dot{q}_{7}\right)^{2}\right) .
\end{aligned}
$$

Using Equation (5) and setting $\mathbf{q}_{\mathrm{cm}}=0$, one can re-write the kinetic energy as:

$$
\begin{aligned}
K= & \frac{M}{3}\left(\dot{q}_{1}^{2}+\left(q_{2} \sin q_{4}\left(\dot{q}_{3}+\dot{q}_{4}\right)-\dot{q}_{2} \cos q_{4}\right) \dot{q}_{1}+\dot{q}_{2}^{2}+\left(q_{1}^{2}-q_{2} q_{1} \cos q_{4}+q_{2}^{2}\right) \dot{q}_{3}^{2}\right. \\
& \left.+q_{2}^{2} \dot{q}_{4}^{2}-q_{1} \dot{q}_{2} \dot{q}_{3} \sin q_{4}+q_{2}\left(2 q_{2}-q_{1} \cos q_{4}\right) \dot{q}_{3} \dot{q}_{4}\right) \\
& +\frac{J}{2}\left(\left(\dot{q}_{3}+\dot{q}_{5}\right)^{2}+\left(\dot{q}_{3}+\dot{q}_{6}\right)^{2}+\left(\dot{q}_{3}+\dot{q}_{4}+\dot{q}_{7}\right)^{2}\right) .
\end{aligned}
$$

\section{Potential Energy}

For spacecraft with sufficiently large nominal inter-satellite distances, magnetic forces between a spacecraft pair can be modeled by a magnetic dipole moment. ${ }^{7}$ In this case, the magnetic potential function is given by

$$
U\left(\mathbf{q}_{1}, \mathbf{q}_{2}\right)=-\boldsymbol{\mu}_{2} \cdot \mathbf{B}_{1}\left(\mathbf{q}_{1}\right)-\boldsymbol{\mu}_{3} \cdot \mathbf{B}_{1}\left(\mathbf{q}_{2}\right)-\boldsymbol{\mu}_{3} \cdot \mathbf{B}_{2}\left(\mathbf{q}_{2}-\mathbf{q}_{1}\right),
$$


where the vector $\boldsymbol{\mu}_{i}$ is the magnetic dipole vector of spacecraft $i, i=1,2,3$, and

$$
\mathbf{B}_{i}(\mathbf{r})=\frac{\mu_{0}}{4 \pi}\left(\frac{3 \mathbf{r}\left(\boldsymbol{\mu}_{i} \cdot \mathbf{r}\right)}{\|\mathbf{r}\|^{5}}-\frac{\boldsymbol{\mu}_{i}}{\|\mathbf{r}\|^{3}}\right)
$$

with $\mu_{0}=4 \pi \times 10^{-7} \mathrm{~Wb} \mathrm{Am}^{-1} \mathrm{~m}^{-1}$ being the permeability in free space. Note that the magnetic potential is a function of both the distance $\|\mathbf{r}\|$ between the two craft, as well as the relative attitude between the dipole moments. Hence, the magnetic potential between two magnets introduces a translational force of attraction/repulsion between the centers of mass of the two craft in one mode of interaction, and also creates a rotational torque between the two magnetic coils in the second mode of interaction. These two modes are illustrated in Figure 2.

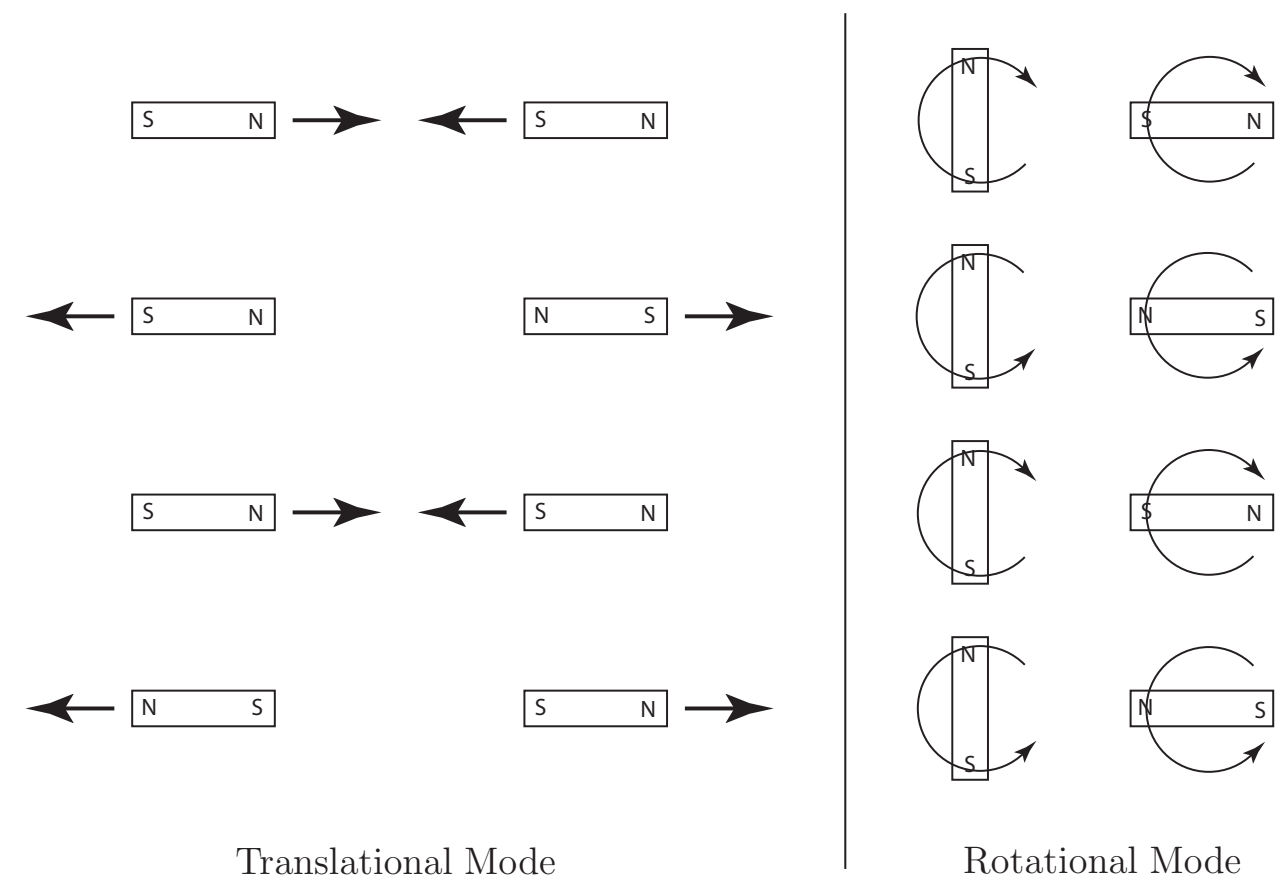

Figure 2. The main magnetic modes of interaction between two magnets.

In coordinates, the far-field magnetic potential energy is given by ${ }^{7}$

$$
\begin{aligned}
U= & -\frac{\left(\cos \left(q_{5}-q_{6}\right)+3 \cos \left(q_{5}+q_{6}\right)\right) \mu_{0} \mu_{1} \mu_{2}}{8 \pi q_{1}^{3}} \\
& -\frac{\mu_{0} \mu_{1} \mu_{3}\left(3 \cos \left(q_{4}-q_{5}-q_{7}\right)+\cos \left(q_{4}-q_{5}+q_{7}\right)\right)}{8 \pi q_{2}^{3}} \\
& -\frac{\left(3 \cos \left(q_{4}-q_{5}-q_{7}\right)+\cos \left(q_{4}-q_{5}+q_{7}\right)\right) \mu_{0} \mu_{1} \mu_{3}}{8 \pi q_{2}^{3}} \\
& \frac{1}{8 \pi\left(q_{1}^{2}-2 q_{2} q_{1} \cos q_{4}+q_{2}^{2}\right)^{\frac{5}{2}}} \\
& \times\left[\left(\left(\cos \left(q_{4}-q_{6}+q_{7}\right)+3 \cos \left(q_{4}+q_{6}+q_{7}\right)\right) q_{1}^{2}-\left(\cos \left(q_{6}-q_{7}\right)+\cos \left(2 q_{4}-q_{6}+q_{7}\right)\right.\right.\right. \\
& \left.\left.\left.+6 q_{2} q_{1} \cos \left(q_{6}+q_{7}\right)\right)+\left(3 \cos \left(q_{4}-q_{6}-q_{7}\right)+\cos \left(q_{4}-q_{6}+q_{7}\right)\right) q_{2}^{2}\right) \mu_{0} \mu_{2} \mu_{3}\right] .
\end{aligned}
$$




\section{E. The Lagrangian, General Equations of Motion, and Conserved Quantities}

The equations of motion are obtained using the Euler-Lagrange equation and the Lagrangian $L=K-U$ :

$$
\begin{aligned}
\frac{\mathrm{d}}{\mathrm{d} t} \frac{\partial L}{\partial \dot{q}_{i}}-\frac{\partial L}{\partial q_{i}} & =0, i=1, \ldots, 4 \\
\frac{\mathrm{d}}{\mathrm{d} t} \frac{\partial L}{\partial \dot{q}_{5}}-\frac{\partial L}{\partial q_{5}} & =\tau_{1} \\
\frac{\mathrm{d}}{\mathrm{d} t} \frac{\partial L}{\partial \dot{q}_{6}}-\frac{\partial L}{\partial q_{6}} & =\tau_{2} \\
\frac{\mathrm{d}}{\mathrm{d} t} \frac{\partial L}{\partial \dot{q}_{7}}-\frac{\partial L}{\partial q_{7}} & =\tau_{3},
\end{aligned}
$$

where $\tau_{1}, \tau_{2}, \tau_{3}$ are three control torque inputs acting on the coils.

Note that the Lagrangian is invariant under rigid body rotations of the overall three-craft system. That is, $L$ is independent of $q_{3}$. Associated with this invariance property is a conservation law that is, namely, the conservation of the generalized angular momentum $h_{3}$ associated with $q_{3}$. The conserved momentum is given by

$$
\begin{aligned}
h_{3}=\frac{\partial L}{\partial \dot{q}_{3}}= & \frac{1}{3}\left(M\left(2 \dot{q}_{3} q_{1}^{2}-q_{2} \cos q_{4}\left(2 \dot{q}_{3}+\dot{q}_{4}\right) q_{1}+\sin q_{4}\left(q_{2} \dot{q}_{1}-q_{1} \dot{q}_{2}\right)+2 q_{2}^{2}\left(\dot{q}_{3}+\dot{q}_{4}\right)\right)\right. \\
& \left.+3 J\left(3 \dot{q}_{3}+\dot{q}_{4}+\dot{q}_{5}+\dot{q}_{6}+\dot{q}_{7}\right)\right) .
\end{aligned}
$$

The infinitesimal generator associated with this group action ${ }^{9}$ is given by $\boldsymbol{\xi}_{G}(\mathbf{q})=(0,0, \xi, 0,0,0,0)$. The vector $\boldsymbol{\xi}_{G}(\mathbf{q})$ is essentially the angular spin rate of the three-craft system about an axis perpendicular to the plane of motion and that passes through our chosen origin at the location of satellite 1.

\section{Relative Equilibria}

To calculate the relative equilibria of the system, we use a geometric approach such as that discussed in detail in Ref. [9]. To do so, we first need to compute the locked inertia tensor, which is given in matrix form by

$$
\mathbb{I}(\mathbf{q})=3 J+\frac{2 M}{3}\left(q_{1}^{2}-q_{1} q_{2} \cos q_{4}+q_{2}^{2}\right) .
$$

The locked inertia tensor is essentially the inertia of the system but with internal shape variables fixed. The "shape variables" are the variables $q_{1}, q_{2}, q_{4}, q_{5}, q_{6}, q_{7}$ that determine the internal shape of the system. If these variables are held fixed, alowing only the overall "group variable" $q_{3}$ to vary, the effective moment of inertia of the system (in this case about spacecraft 1) is that given by $\mathbb{I}$ above. With this, one computes the 
augmented potential function ${ }^{9} U_{\boldsymbol{\xi}}$ :

$$
\begin{aligned}
U_{\boldsymbol{\xi}}= & U-\frac{1}{2} \xi^{2} \mathbb{I} \\
= & -\frac{\left(\cos \left(q_{5}-q_{6}\right)+3 \cos \left(q_{5}+q_{6}\right)\right) \mu_{0} \mu_{1} \mu_{2}}{8 \pi q_{1}^{3}} \\
& -\frac{\mu_{0} \mu_{1} \mu_{3}\left(3 \cos \left(q_{4}-q_{5}-q_{7}\right)+\cos \left(q_{4}-q_{5}+q_{7}\right)\right)}{8 \pi q_{2}^{3}} \\
& -\frac{\left(3 \cos \left(q_{4}-q_{5}-q_{7}\right)+\cos \left(q_{4}-q_{5}+q_{7}\right)\right) \mu_{0} \mu_{1} \mu_{3}}{8 \pi q_{2}^{3}} \\
& \frac{1}{8 \pi\left(q_{1}^{2}-2 q_{2} q_{1} \cos q_{4}+q_{2}^{2}\right)^{\frac{5}{2}}} \\
& \times\left[\left(\left(\cos \left(q_{4}-q_{6}+q_{7}\right)+3 \cos \left(q_{4}+q_{6}+q_{7}\right)\right) q_{1}^{2}-\left(\cos \left(q_{6}-q_{7}\right)+\cos \left(2 q_{4}-q_{6}+q_{7}\right)\right.\right.\right. \\
& \left.\left.\left.+6 q_{2} q_{1} \cos \left(q_{6}+q_{7}\right)\right)+\left(3 \cos \left(q_{4}-q_{6}-q_{7}\right)+\cos \left(q_{4}-q_{6}+q_{7}\right)\right) q_{2}^{2}\right) \mu_{0} \mu_{2} \mu_{3}\right] \\
& -\frac{1}{2} \xi^{2}\left[3 J+\frac{2 M}{3}\left(q_{1}^{2}-q_{1} q_{2} \cos q_{4}+q_{2}^{2}\right)\right]
\end{aligned}
$$

If there exist $\xi$ such that

$$
\left.\frac{\partial U_{\boldsymbol{\xi}}}{\partial q_{i e}}\right|_{e}=0, i=1,2,4,5,6,7,
$$

where the subscript $e$ signifies evaluating the expression at the equilibrium point of interest, hold true, then $\left(q_{1 e}, q_{2 e}, q_{4 e}, q_{5 e}, q_{6 e}, q_{7 e}\right)$ is a relative equilibrium. This condition is necessary and sufficient. In other words all solutions to the nonlinear algebraic Equations (11) are relative equilibria and any solution that satisfies these conditions is a relative equilibrium. We omit the explicit expressions of Equation (11) due to the length of the resulting equations. Finding all solutions of these equations is a challenging task computationally and we do not perform such computations in this paper. In this paper we postulate 3 relative equilibrium solutions (shown in Figure 3) that they satisfy the above conditions.

\section{A. Relative Equilibrium 1: Aligned Configuration}

The first equilibrium is one where all three coils are aligned such that the south pole of one coil is positioned facing the north pole of another coil, and vice versa. This relative equilibrium is postulated because the alternating arrangement of the coils results in an overall attractive force. Since this force would result in the collapse of the coils to the center of mass of the system if no centripetal force is introduced, one then expects that the system needs to be spun at a specific angular rate that produces enough centripetal force to counteract the attractive forces generated by the magnets. We first express the values of the configuration variables at the equilibrium:

$$
\begin{aligned}
& q_{1}=q_{*} \\
& q_{2}=2 q_{*} \\
& q_{4}=q_{5}=q_{6}=q_{7}=0 \\
& \dot{q}_{4}=\dot{q}_{5}=\dot{q}_{6}=\dot{q}_{7}=0,
\end{aligned}
$$

where $q_{*}$ is the relative distance between coils 1 and 2, and then use Equation (11) to derive conditions on the dipole moments and the spin rate $\xi$ at equilibrium. These turn out to be:

$$
\begin{aligned}
\mu_{1}^{*} & =\mu_{3}^{*} \\
\xi & =\sqrt{\frac{3 \mu_{0}\left(\mu_{1}^{*}+16 \mu_{2}^{*}\right) \mu_{1}^{*}}{32 M \pi q_{*}^{5}}} .
\end{aligned}
$$


Note that the first condition expresses the fact that if there is an imbalance in the dipole moment of the two outer-most coils, there is an overall net force acting towards one end of the system that destroys steady equilibrium conditions. Note also that there are no conditions on the strength of the central dipole moment $\mu_{2}^{*}$. This is because if $\mu_{2}^{*}$ is too large compared to $\mu_{1}^{*}=\mu_{3}^{*}$, this will be remedied by requiring that $\xi=\left(\dot{q}_{3}\right)_{e}$ be larger to supply enough centripetal force to counteract the strength of the central magnet. If $\mu_{2}^{*}$ is too small, say zero, the system is composed of only two attracting magnets and the analysis reduces to that provided in Ref. [8]. In that case, the spin rate $\xi$ is equal to the exact spin required to produce a centripetal force equal to the attractive force of attraction between the two magnets. The equilibrium momentum can be computed from Equation (9). At equilibrium, the conserved momentum is given by

$$
h_{3}=\left(3 J+2 M q_{*}^{2}\right) \xi .
$$

\section{B. Relative Equilibrium 2: Parallel Configuration}

The second equilibrium is one where all three coils are arranged parallel to each other such that the south pole of one coil is positioned next to the north pole of another coil, and vice versa. This relative equilibrium is postulated because the alternating arrangement of the coils results in an overall attractive force. Since this force would result in the collapse of the coils to the center of mass of the system if no centripetal force is introduced, one then expects that the system needs to be spun at a specific angular rate that produces enough centripetal force to counteract the attractive forces generated by the magnets. We first express the values of the configuration variables at the equilibrium:

$$
\begin{aligned}
& q_{1}=q_{*} \\
& q_{2}=2 q_{*} \\
& q_{4}=0 \\
& q_{5}=-q_{6}=q_{7}=\frac{\pi}{2} \\
& \dot{q}_{4}=\dot{q}_{5}=\dot{q}_{6}=\dot{q}_{7}=0,
\end{aligned}
$$

where $q_{*}$ is the relative distance between coils 1 and 2, and then use Equation (11) to derive conditions on the dipole moments and the spin rate $\xi$ at equilibrium. These turn out to be:

$$
\begin{aligned}
\mu_{1}^{*} & =\mu_{3}^{*} \\
\xi & =\sqrt{-\frac{3 \mu_{0}\left(\mu_{1}^{*}-16 \mu_{2}^{*}\right) \mu_{1}^{*}}{64 M \pi r^{5}}} .
\end{aligned}
$$

Note that the first condition, as with the first equilibrium, expresses the fact that if there is an imbalance in the dipole moment of the two outer-most coils, there is an overall net force acting towards one end of the system that destroys steady equilibrium conditions. Note, however, that there is a condition on the strength of the center dipole moment $\mu_{2}^{*}$. If

$$
\mu_{2}^{*}<\frac{1}{16} \mu_{1}^{*}
$$

then $\xi$ will assume imaginary values, which is not allowed physically. To see why this condition on $\xi_{2}$ arise physically, note that if $\mu_{2}^{*}$ is sufficiently small, assume zero without any loss of generality, the two outer-most magnets will repel (hence, causing an unbounded expansion of the system and, thus, violating the definition of an equilibrium) and no spin rate can every be applied to retain the equilibrium. According to the above calculation, the smallest value that $\mu_{2}^{*}$ can assume is $\mu_{1}^{*} / 16=\mu_{3}^{*} / 16$. At this critical value, the required spin rate is exactly $\xi=0$, which corresponds to a static (i.e., non-spinning) equilibrium. The equilibrium momentum can be computed from Equation (9). At equilibrium, the conserved momentum is given by

$$
h_{3}=\left(3 J+2 M q_{*}^{2}\right) \xi .
$$

\section{Relative Equilibrium 3: Circular Configuration}

The third equilibrium is one where the three coils are arranged in a circular configuration centered at the center of mass of the system, and such that the south pole of one coil is pointing towards the north pole of 
another coil, and vice versa. Each coil is aligned tangent to the circle. This relative equilibrium is postulated because the alternating arrangement of the coils results in an overall attractive force towards the center of mass of the system. Since this force would result in the collapse of the coils to the center of mass of the system if no centripetal force is introduced, one then expects that the system needs to be spun at a specific angular rate that produces enough centripetal force to counteract the attractive forces generated by the magnets. We first express the values of the configuration variables at the equilibrium:

$$
\begin{aligned}
q_{1} & =q_{*} \\
q_{2} & =q_{*} \\
q_{4} & =\frac{\pi}{3} \\
q_{5} & =-q_{6}=\frac{1}{2} q_{7}=\frac{\pi}{3} \\
\dot{q}_{4} & =\dot{q}_{5}=\dot{q}_{6}=\dot{q}_{7}=0,
\end{aligned}
$$

where $q_{*}$ is the relative distance between coils 1 and 2, 1 and 3, and 2 and 3, and then use Equation (11) to derive conditions on the dipole moments and the spin rate $\xi$ at equilibrium. These turn out to be:

$$
\begin{aligned}
\mu_{1}^{*} & =\mu_{2}^{*}=\mu_{3}^{*} \\
\xi & =\sqrt{\frac{45 \mu_{0}\left(\mu_{1}^{*}\right)^{2}}{16 M \pi r^{5}}} .
\end{aligned}
$$

Note that the three dipole moments need to be equal for the existence of a circular relative equilibrium, otherwise there will be a net force that is not directed towards the center of mass of the system, which would disturb the system from equilibrium. The equilibrium momentum can be computed from Equation (9). At equilibrium, the conserved momentum is given by

$$
h_{3}=\left(3 J+M q_{*}^{2}\right) \xi .
$$

Note that this equilibrium is slightly different from that computed above for the first two equilibria. This is because the mass distribution in this equilibrium configuration is different from that in the first two equilibria.

As an illustration, Figure 4 shows the trajectories for the variables $\left(q_{1}, \ldots, q_{7}\right)$ as well as $\dot{q}_{3}$ for the third equilibrium. The parameters chosen for the simulation are as follows

$$
M=1 \mathrm{Kg}, J=1 \mathrm{Kg} \mathrm{m}^{2}, q_{*}=1 \mathrm{~m}, \mu_{1}^{*}=\mu_{2}^{*}=\mu_{3}^{*}=1 \times 10^{5} \mathrm{~A} \mathrm{~m}^{2},
$$

with initial conditions set such that the equilibrium conditions in Equations (18) are satisfied and zero external torques $\tau_{1}, \tau_{2}, \tau_{3}$. The spin rate for these parameters is given by $\xi=6077.14$ degrees per second. As can be seen from the plots, the system is verified to be in equilibrium. The solution was obtained numerical by integrating the Euler-Lagrange equations using NDSolve command in Mathemaica ${ }^{\circledR}$. The duration of the simulation is 5 hours (18,000 seconds).

\section{Reduced Equations of Motion}

We will use the conserved angular momentum $h_{3}$ to reduce the equations of motion using Routhian reduction. $^{9}$ In the next section, these equations will be linearized to determined stability of one of the identified relative equilibria. The Routhian is defined as

$$
R\left(q_{1}, q_{2}, q_{4}, q_{5}, q_{6}, q_{7}, \dot{q}_{1}, \dot{q}_{2}, \dot{q}_{4}, \dot{q}_{5}, \dot{q}_{6}, \dot{q}_{7}\right)=\left[L-h_{3} \dot{q}_{3}\right]
$$

with $\dot{q}_{3}$ solved for and substituted using Equations (14), (17), or (20) depending on the equilibrium being considered. Hence $R$ is independent of $q_{3}$ and its derivatives. The Routhian function $R$ is shown in Figure 5 . 


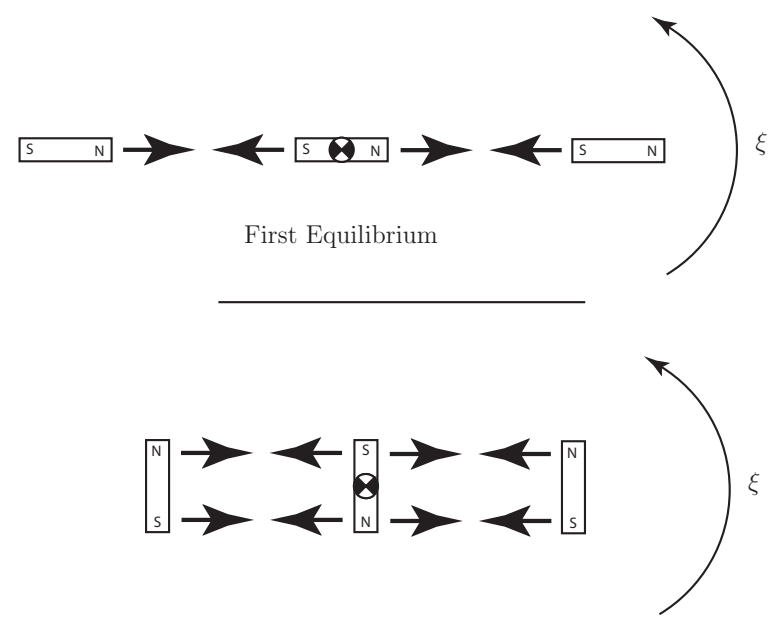

Second Equilibrium

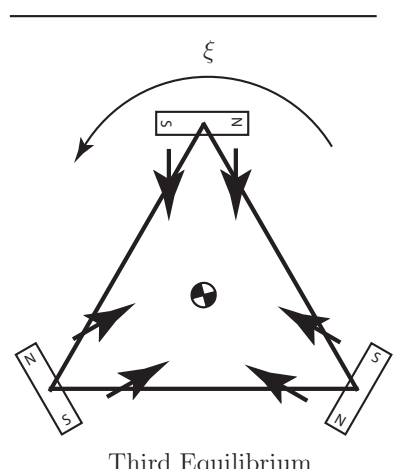

Third Equilibrium

Figure 3. The three postulated equilibria. Other equilibria may exist.

The reduced equations of motion are then given by

$$
\begin{aligned}
& \frac{\mathrm{d}}{\mathrm{d} t} \frac{\partial R}{\partial \dot{q}_{1}}-\frac{\partial R}{\partial q_{1}}=0 \\
& \frac{\mathrm{d}}{\mathrm{d} t} \frac{\partial R}{\partial \dot{q}_{2}}-\frac{\partial R}{\partial q_{2}}=0 \\
& \frac{\mathrm{d}}{\mathrm{d} t} \frac{\partial R}{\partial \dot{q}_{4}}-\frac{\partial R}{\partial q_{4}}=0 . \\
& \frac{\mathrm{d}}{\mathrm{d} t} \frac{\partial R}{\partial \dot{q}_{5}}-\frac{\partial R}{\partial q_{5}}=\tau_{1} \\
& \frac{\mathrm{d}}{\mathrm{d} t} \frac{\partial R}{\partial \dot{q}_{6}}-\frac{\partial R}{\partial q_{6}}=\tau_{2} \\
& \frac{\mathrm{d}}{\mathrm{d} t} \frac{\partial R}{\partial \dot{q}_{7}}-\frac{\partial R}{\partial q_{7}}=\tau_{3}
\end{aligned}
$$

These equations are a few pages long when expanded and, hence, we do not show them in this paper. These equations will be used in the next sections to evaluate the stability and controllability for one of the relative equilibria. Linearization based control techniques will then be used to control the three-craft system about that equilibrium. 


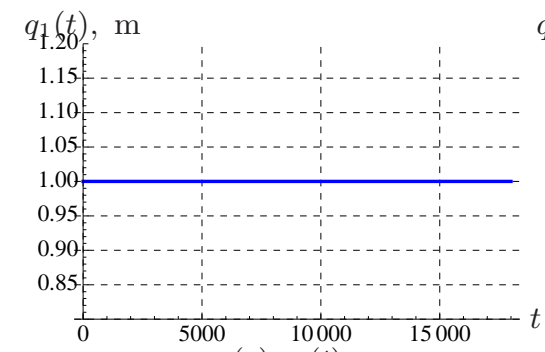

(a) $q_{1}(t)$

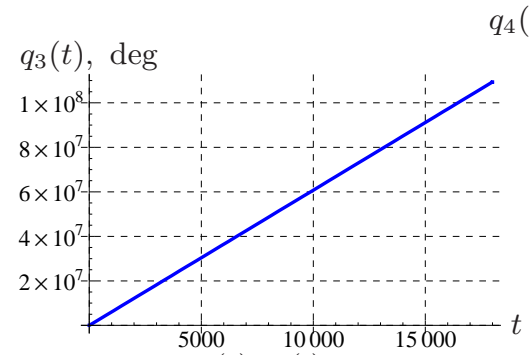

(c) $q_{3}(t)$

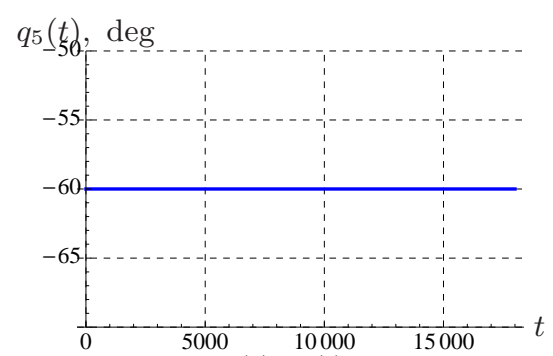

(e) $q_{5}(t)$

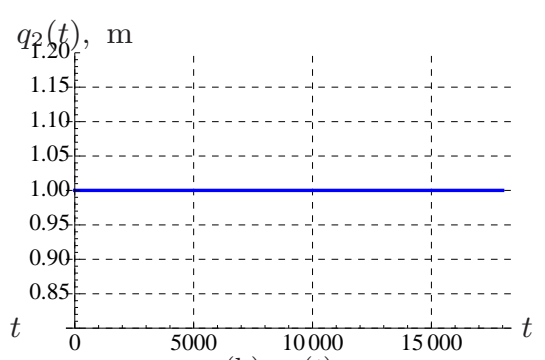

(b) $q_{2}(t)$

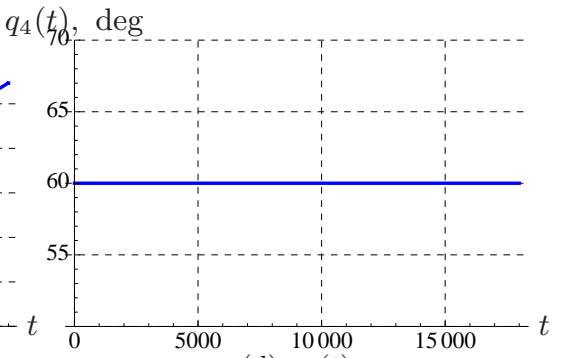

(d) $q_{4}(t)$

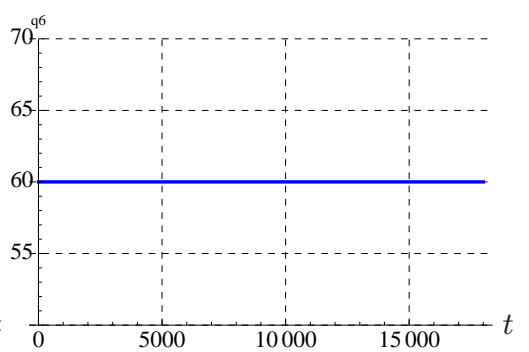

(f) $q_{6}(t)$

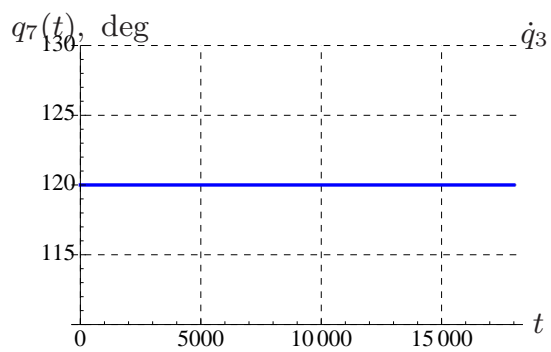

(g) $q_{7}(t)$

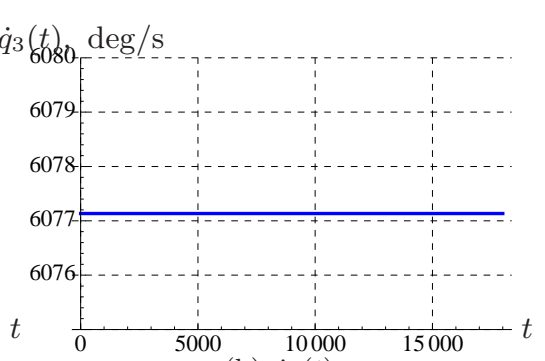

(h) $\dot{q}_{3}(t)$

Figure 4. Simulation results verifying the conditions for the third equilibrium. First and second equilibria can be verified as well (not shown).

\section{Stability of the Relative Equilibria}

Linearized Equations. The reduced Equations (23) can be linearized and the characteristic polynomial computed. The linearized equations (about nominal control variables $\mu_{1}=\mu_{1}^{*}, \mu_{2}=\mu_{2}^{*}, \mu_{3}=\mu_{3}^{*}, \tau_{1}=\tau_{2}=$ $\tau_{3}=0$ and a specific equilibrium condition) have the form:

$$
\mathbf{A} \ddot{\mathbf{x}}+\mathbf{B} \dot{\mathbf{x}}+\mathbf{C x}=0
$$




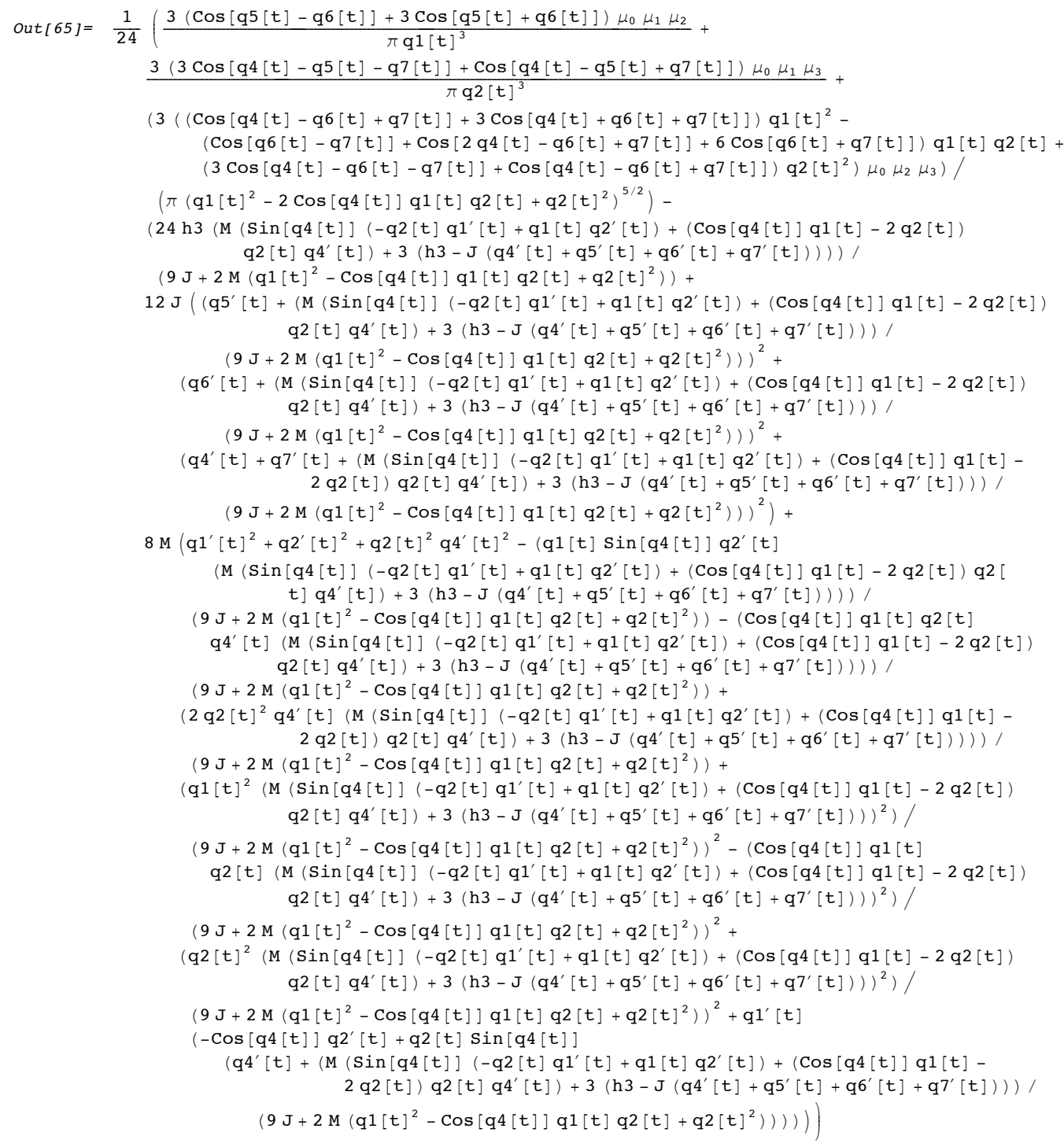

Figure 5. A Mathematica output for the system Routhian function. 
where the matrices are evaluated at the desired equilibrium, and with

$$
\mathbf{x}(t)=\left[\begin{array}{c}
q_{1}(t) \\
q_{2}(t) \\
q_{4}(t) \\
q_{5}(t) \\
q_{6}(t) \\
q_{7}(t)
\end{array}\right] .
$$

In this paper we derive the matrices $\mathbf{A}, \mathbf{B}$ and $\mathbf{C}$ (and later for the control input matrix $\mathbf{D}$ and the momentum matrix coefficient $\mathbf{E}$ ) for the third equilibrium only. The process can be repeated for any other equilibrium of the system. The matrices for the third equilibrium are given by

$$
\mathbf{A}=\left[\begin{array}{cccccc}
\frac{M}{12}\left(\frac{3 J}{\kappa}+7\right) & -\frac{M}{12}\left(\frac{3 J}{\kappa}+1\right) & \frac{M q_{*} \beta}{4 \sqrt{3} \kappa} & -\frac{J M q_{*}}{2}{ }^{3} \kappa & -\frac{J M q_{*}}{2 \sqrt{3} \kappa} & -\frac{J M q_{*}}{2 \sqrt{3} \kappa} \\
\frac{M}{12}\left(-\frac{3 J}{\kappa}-1\right) & \frac{M}{12}\left(\frac{3 J}{\kappa}+7\right) & \frac{M q_{*} \alpha}{4 \sqrt{3} \kappa} & \frac{J M q_{*}}{2 \sqrt{3} \kappa} & \frac{J M q_{*}}{2 \sqrt{3} \kappa} & \frac{J M q_{*}}{2 \sqrt{3} \kappa} \\
\frac{M q_{*} \beta}{4 \sqrt{3} \kappa} & \frac{M q_{*} \alpha}{4 \sqrt{3} \kappa} & \frac{1}{12}\left(-\frac{3 J^{2}}{\kappa}+9 J+5 M q_{*}^{2}\right) & -\frac{J \alpha}{2 \kappa} & -\frac{J \alpha}{2 \kappa} & \frac{J \beta}{2 \kappa} \\
-\frac{J M q_{*}}{2 \sqrt{3} \kappa} & \frac{J M q_{*}}{2 \sqrt{3} \kappa} & -\frac{J \alpha}{2 \kappa} & J-\frac{J^{2}}{\kappa} & -\frac{J^{2}}{\kappa} & -\frac{J^{2}}{\kappa} \\
-\frac{J M q_{*}}{2 \sqrt{3} \kappa} & \frac{J M q_{*}}{2 \sqrt{3} \kappa} & -\frac{J \alpha}{2 \kappa} & -\frac{J^{2}}{\kappa} & J-\frac{J^{2}}{\kappa} & -\frac{J^{2}}{\kappa} \\
-\frac{J M q_{*}}{2 \sqrt{3} \kappa} & \frac{J M q_{*}}{2 \sqrt{3} \kappa} & \frac{J \beta}{2 \kappa} & -\frac{J^{2}}{\kappa} & -\frac{J^{2}}{\kappa} & J-\frac{J^{2}}{\kappa}
\end{array}\right]
$$

where $\kappa=M q_{*}^{2}+3 J, \alpha=M q_{*}^{2}+2 J$ and $\beta=M q_{*}^{2}+4 J$,

$$
\mathbf{B}=\left[\begin{array}{cccccc}
0 & \frac{\sqrt{3} J M h_{3}}{\kappa^{2}} & \frac{2 M q_{*} h_{3}}{3 \kappa} & \frac{J M q_{*} h_{3}}{\kappa^{2}} & \frac{J M q_{*} h_{3}}{\kappa^{2}} & \frac{J M q_{*} h_{3}}{\kappa^{2}} \\
-\frac{\sqrt{3} J M h_{3}}{\kappa^{2}} & 0 & -\frac{M q_{*} \gamma h_{3}}{3 \kappa^{2}} & \frac{J M q_{*} h_{3}}{\kappa^{2}} & \frac{J M q_{*} h_{3}}{\kappa^{2}} & \frac{J M q_{*} h_{3}}{\kappa^{2}} \\
-\frac{2 M q_{*} h_{3}}{3 \kappa} & \frac{M q_{*} \gamma h_{3}}{3 \kappa^{2}} & 0 & \frac{J M q_{*}^{2} h_{3}}{\sqrt{3} \kappa^{2}} & \frac{J M q_{*}^{2} h_{3}}{\sqrt{3} \kappa^{2}} & \frac{J M q_{*}^{2} h_{3}}{\sqrt{3} \kappa^{2}} \\
-\frac{J M q_{*} h_{3}}{\kappa^{2}} & -\frac{J M q_{*} h_{3}}{\kappa^{2}} & -\frac{J M q_{*}^{2} h_{3}}{\sqrt{3} \kappa^{2}} & 0 & 0 & 0 \\
-\frac{J M q_{*} h_{3}}{\kappa^{2}} & -\frac{J M q_{*} h_{3}}{\kappa^{2}} & -\frac{J M q_{*}^{2} h_{3}}{\sqrt{3} \kappa^{2}} & 0 & 0 & 0 \\
-\frac{J M q_{*} h_{3}}{\kappa^{2}} & -\frac{J M q_{*} h_{3}}{\kappa^{2}} & -\frac{J M q_{*}^{2} h_{3}}{\sqrt{3} \kappa^{2}} & 0 & 0 & 0
\end{array}\right],
$$

where $\gamma=2 M q_{*}^{2}+9 J$, and with

$\mathrm{C}=$
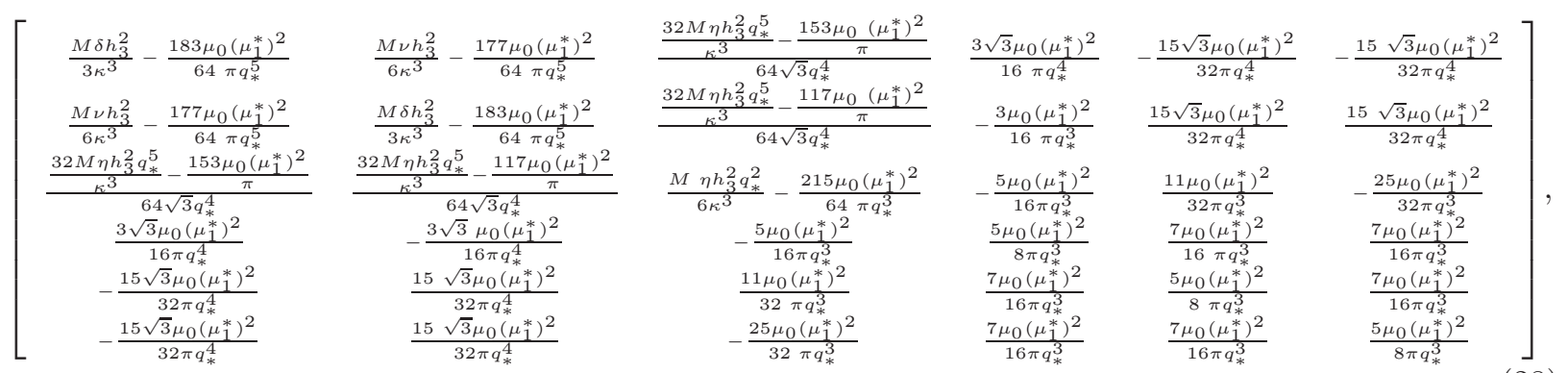

where $\eta=M q_{*}^{2}-3 J, \delta=M q_{*}^{2}-6 J, \nu=7 M q_{*}^{2}+3 J$, and where we have substituted for the conditions for relative equilibrium (thus, for example, substituting $\mu_{2}^{*}, \mu_{3}^{*}$ with $\mu_{1}^{*}$ ).

It is anticipated that all equilibria of the system are unstable. This is because magnetic systems are generally unstable if no damping effects act on the system. However, proving general instability for a given equilibrium is difficult to do because the expressions for the eigenvalues of the linearized system are very complex to analyze. Therefore, in this paper we demonstrate that third equilibrium is unstable for a specific choice of the parameters. Similar computations can be performed for the other two equilibria.

Substituting for the system parameters in the above matrices using the values introduced in Equation (21), the system characteristic polynomial is found to be

$$
\begin{aligned}
p(s)= & 0.0277778 s^{12}-1304.61 s^{10}+19.0638 s^{9}-1.58988 \times 10^{7} s^{8}-486068 . s^{7}+7.94624 \times 10^{11} s^{6} \\
& +2.39918 \times 10^{10} s^{5}-3.90087 \times 10^{14} s^{4}+1.5762 \times 10^{14} s^{3}-3.58139 \times 10^{19} s^{2}+1.6968 \times 10^{17} s \\
& -7.37564 \times 10^{22} .
\end{aligned}
$$


Solving $p(s)=0$, one obtains the system eigenvalues, which are

$$
\begin{aligned}
\lambda_{1} & =-215.56, \lambda_{2}=-149.74, \lambda_{3}=-95.98, \lambda_{4,5}=-0.0018 \pm 49.76 \imath \\
\lambda_{6,7} & =-0.00031 \pm 68.23 \imath, \lambda_{8,9}=0.0092 \pm 154.92 \imath, \lambda_{10}=95.93, \lambda_{11}=149.8, \lambda_{12}=215.54 .
\end{aligned}
$$

Clearly, the system is unstable about the third equilibrium for the chosen set of parameters. Of course this does not imply that the equilibrium is generally unstable for there may exist other parameter choices that renders the system stable about the third equilibrium. This scenario is highly unlikely for magnetic systems without damping. Determining stability or lack thereof for three-craft systems is the focus of future work. To demonstrate lack of stability numerically, Figure 6 shows a simulation for the above chosen parameters. Instead of initializing the system exactly at the equilibrium values, we perturb the initial spin rate from its nominal value of 6077.14 degrees per second to 6077.19 degrees per second. Notice that even a very small change in the spin rate (a change of $0.0009 \%$ ) results in a very large error in a very short amount of time. This indicates that a magnetic tether system is very sensitive to errors. Stabilizing the system about the third equilibrium is the subject of the next two sections.

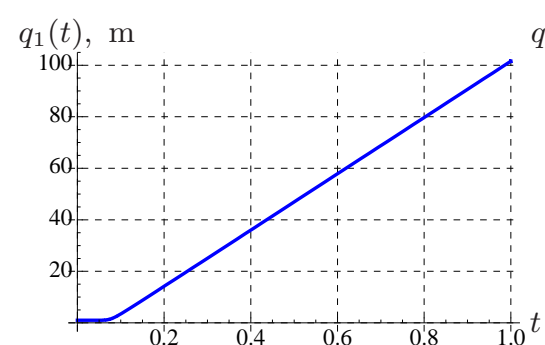

(a) $q_{1}(t)$

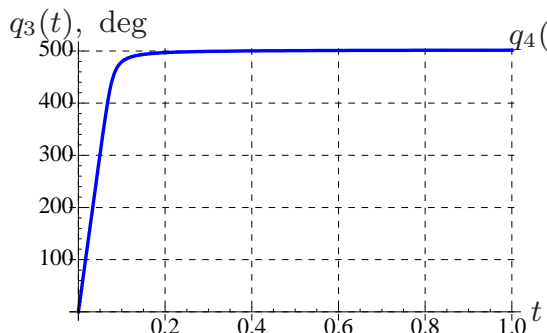

(c) $q_{3}(t)$

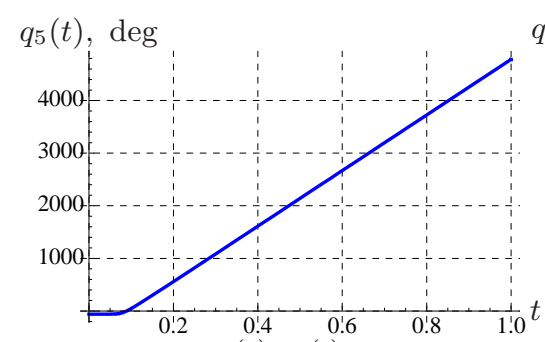

(e) $q_{5}(t)$

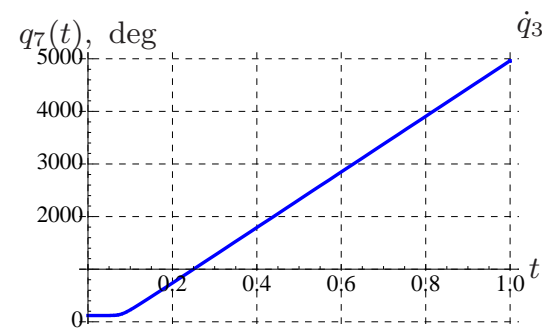

(g) $q_{7}(t)$

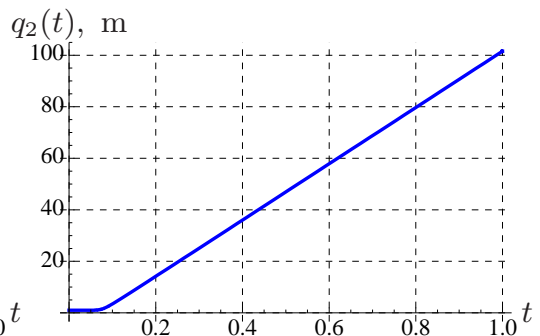

(b) $q_{2}(t)$

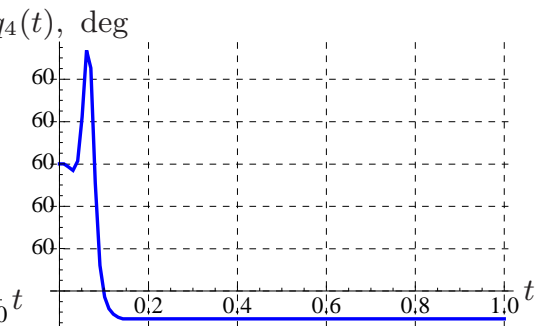

(d) $q_{4}(t)$

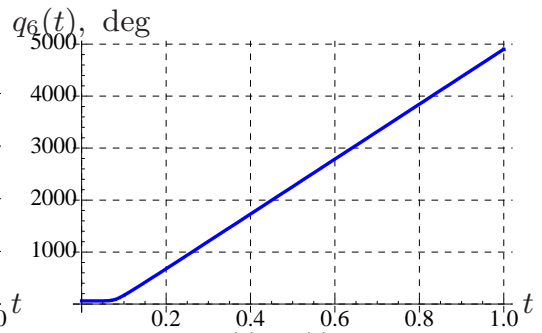

(f) $q_{6}(t)$

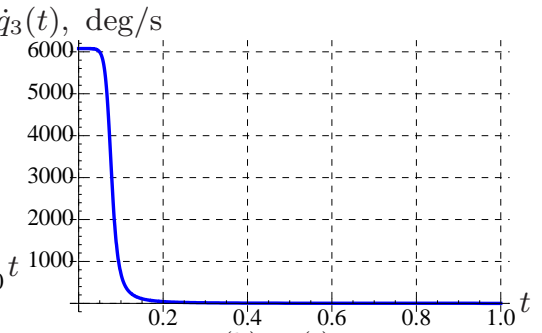

(h) $\dot{q}_{3}(t)$

Figure 6. Simulation results verifying the instability of the system about the third equilibrium for the set of chosen parameter values. 


\section{Controllability}

Lets consider using $\left(\mu_{1}, \mu_{2}, \mu_{3}, \tau_{1}, \tau_{2}, \tau_{3}\right)$ as control inputs. The equations of motion will then take the form:

$$
\mathbf{A} \ddot{\mathbf{x}}+\mathbf{B} \dot{\mathbf{x}}+\mathbf{C x}=\mathbf{D u}
$$

Again, due to the complexity of the equations we will only give a numerical example to study the controllability of the system near the third equilibrium. For the third equilibrium, we have

$$
=\left[\begin{array}{cccccc}
\frac{15 \mu_{0} \mu_{1}^{*}}{16 \pi q_{*_{1}^{4}}^{4}} & \frac{45 \mu_{0} \mu_{1}^{*}}{32 \pi q_{1}^{4}} & \frac{15 \mu_{0} \mu_{1}^{*}}{32 \pi q_{\psi_{*}^{*}}^{4}} & 0 & 0 & 0 \\
\frac{15 \mu_{0}}{16 \pi q_{*}^{4}} & \frac{15 \mu_{0} \mu_{1}^{4}}{32 \pi q_{*}^{4}} & \frac{45 \mu_{0} \mu_{1}^{4}}{32 \pi q_{*}^{4}} & 0 & 0 & 0 \\
-\frac{\sqrt{3} \mu_{0} \mu_{1}^{*}}{16 \pi q_{*}^{3}} & \frac{17 \sqrt{3} \mu_{0} \mu_{1}^{*}}{32 \pi q_{*}^{3}} & \frac{15 \sqrt{3} \mu_{0} \mu_{1}^{*}}{32 \pi q_{*}^{3}} & 0 & 0 & 0 \\
0 & -\frac{\sqrt{3} \mu_{0} \mu_{1}^{*}}{16 \pi q_{*}^{3}} & \frac{\sqrt{3} \mu_{0} \mu_{1}^{*}}{16 \pi q_{*}^{3}} & 1 & 0 & 0 \\
\frac{\sqrt{3} \mu_{0} \mu_{1}^{*}}{16 \pi q_{*}^{3}} & 0 & -\frac{\sqrt{3} \mu_{0} \mu_{1}^{*}}{16 \pi q_{*}^{3}} & 0 & 1 & 0 \\
-\frac{\sqrt{3} \mu_{0} \mu_{1}^{*}}{16 \pi q_{*}^{3}} & \frac{\sqrt{3} \mu_{0} \mu_{1}^{*}}{16 \pi q_{*}^{3}} & 0 & 0 & 0 & 1
\end{array}\right] .
$$

The control input is given by

$$
\mathbf{u}=\left[\begin{array}{c}
\mu_{1}(t) \\
\mu_{2}(t) \\
\mu_{3}(t) \\
\tau_{1}(t) \\
\tau_{2}(t) \\
\tau_{3}(t)
\end{array}\right]
$$

The above linearization assumes that the angular momentum is conserved under any perturbations in initial conditions. To relax this assumption we have to include an additional term in the linearized equations that takes into account perturbations in $h_{3}$. We will comment on this question later in the paper.

The shape dynamics are controllable using the controls $\left(\mu_{1}, \mu_{2}, \mu_{3}, \tau_{1}, \tau_{2}, \tau_{3}\right)$ if and only if

$$
\operatorname{rank}\left(\left[s^{2} \mathbf{A}_{e i}+s \mathbf{B}_{e i}+\mathbf{C}_{e i} \quad \mathbf{D}_{e i}\right]\right)=6
$$

holds for all eigenvalues $s$ that satisfy $\operatorname{det}\left(s^{2} \mathbf{A}_{e i}+s \mathbf{B}_{e i}+\mathbf{C}_{e i}\right)=0 .{ }^{10,11}$ The right hand side has to be 6 because this is the dimension of the reduced shape dynamics. As in previous sections, we will check this condition assuming we desire to stabilize the third equilibrium and assuming the same parameter values as above. Checking the controllability rank condition, one finds that the rank is in fact 6 for all eigenvalues listed in Equation (30), which implies that the reduced equations of motion (i.e., shape dynamics) are controllable if one uses $\left(\mu_{1}, \mu_{2}, \mu_{3}, \tau_{1}, \tau_{2}, \tau_{3}\right)$ as control inputs. In the next section we will use linearization-based feedback control techniques to stabilize the third equilibrium for the above choice of system parameters.

\section{Linearization-Based Feedback Control}

In this section we will use classical quadratic linear feedback control to stabilize the shape dynamics assuming that perturbations do not cause a disturbance in the value of the momentum $h_{3}$. If the perturbations affect the configuration and velocity variables of the system without modifying the conserved momentum $h_{3}$, then a controller design procedure that ignores perturbations in the momentum is acceptable. In this case the linearized equation of motion is given by Equation (31). A linear feedback control law that stabilizes the linearized equations asymptotically will stabilize the nonlinear shape dynamics locally.

However, in general, the perturbation forces will also affect the momentum. In this case, the linearization process has to take into account the fact that the momentum $h_{3}$ can also change. Under this scenario, a new term, $\mathbf{E} \delta h_{3}$, in the linearization will arise and will have to be accounted for. We return to this question later in the section. 


\section{A. Numerical Simulation with zero perturbations in momenta}

Consider the problem of stabilizing the third equilibrium. As we saw the third equilibrium is controllable and hence are also stabilizable. In this section we use optimal linear quadratic regulation to derive a feedback gain matrix $\mathbf{K}_{1}$ to stabilize the system. For the sake of the simulation, we will use the system parameters given in Equation (21). Given the value of $q_{*}$ and choice of nominal dipole moments, we obtain (from Equation (19)) $\xi=6077.14$ degrees per second. The corresponding value of the momentum $h_{3}$ is $h_{3}=424.264 \mathrm{Kg}$ $\mathrm{m} / \mathrm{sec}^{2}$. For initial conditions we perturb $q_{1}$ to a value of $q_{10}=0.9 \mathrm{~m}$, and with $q_{40}=61^{\circ}$ and $q_{50}=-58^{\circ}$ with all other configuration and velocity variables matching the nominal equilibrium values. However, we desire to maintain $h_{2}$ unperturbed from the nominal value $h_{3}=424.264 \mathrm{Kg} \mathrm{m} / \mathrm{sec}^{2}$. In order to do that, we have to reduce the value of $\xi$ (to maintain a constant value of $h_{3}$ ) to become $\xi=6207.84$ degrees per second. The $\mathbf{Q}$ and $\mathbf{R}$ matrices used to obtain an LQR-based feedback gain matrix $\mathbf{K}$ are:

$$
\mathbf{Q}=\operatorname{diag}(5,5,45,45,45,45,0.01,0.01,0.01,0.01,0.01,0.01), \mathbf{R}=\operatorname{diag}\left(10^{-10}, 10^{-10}, 10^{-10}, 1,1,1\right),
$$

where the state in first order form is given by $\tilde{\mathbf{x}}=\left[\begin{array}{l}\mathbf{x} \\ \mathbf{x}\end{array}\right]^{T} \in \mathbb{R}^{1} 2$ (i.e., shape variables only). Using Mathematica ${ }^{\mathbb{B}}$ 's NDSolve and a simulation time range of 5 hours, the results are shown in Figure 7. Since the initial conditions satisfy the nominal momentum value, we expect that the system is stabilized to the desired nominal equilibrium condition. Figure 8 shows the control inputs $\left(\mu_{1}, \mu_{2}, \mu_{3}, \tau_{1}, \tau_{2}, \tau_{3}\right)$. Note that the control inputs converge to their corresponding nominal values.

\section{B. A Comment on Perturbations with nonzero change in momentum}

Finally, we make a comment on the case where the perturbation force causes a change $\delta h_{3}$ in the momentum $h_{3}$. In this case the linearized equations of motion are given by

$$
\mathbf{A} \ddot{\mathbf{x}}+\mathbf{B} \dot{\mathbf{x}}+\mathbf{C x}=\mathbf{D u}+\mathbf{E} \delta h_{3},
$$

where

$$
\mathbf{E}=\left[\begin{array}{c}
-\frac{h_{3} M q_{*}}{\kappa^{2}} \\
-\frac{h_{3} M q_{*}}{\kappa^{2}} \\
-\frac{h_{3} M q_{*}^{2}}{\sqrt{3} \kappa^{2}} \\
0 \\
0 \\
0
\end{array}\right]
$$

for the third equilibrium, for example.

If we assume a feedback control of the form $\mathbf{u}=-\mathbf{K x}$, then, due to the constant error $\delta h_{3}$, the steady state error will be

$$
\mathbf{x}_{s s}=(\mathbf{C}+\mathbf{D K})^{-1} \mathbf{E} \delta h_{3} .
$$

As we see, if the perturbations cause a nonzero change in $h_{3}$, the system will never asymptotically stabilize to the desired nominal value but, instead, to an orbit whose angular momentum is given by the value of $h_{3}$ evaluated based on the erroneous initial conditions. In the case when perturbations do change the angular momentum level, one can apply PID-type controllers, similar to the application of PID control for the 3-craft Coulomb tether problem discussed in Ref. [5] for Coulomb tether systems. We refer the reader to that work as a similar approach can be applied to magnetic tether systems that we discuss in this paper.

\section{Conclusion}

In this paper we studied the dynamics of a simple three-spacecraft formation, where the inter-satellite relative motion is controlled though magnetic coils, each on a different spacecraft, and torques acting on the coils using motors mounted on the spacecraft bodies. Relative equilibrium conditions were derived and

three solutions to these conditions were proposed. Given the complexity of the equations, we used a choice of system parameters to demonstrate that the third equilibrium is unstable for the choice of parameters, 


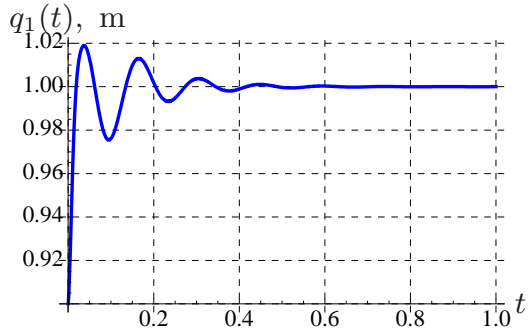

(a) $q_{1}(t)$

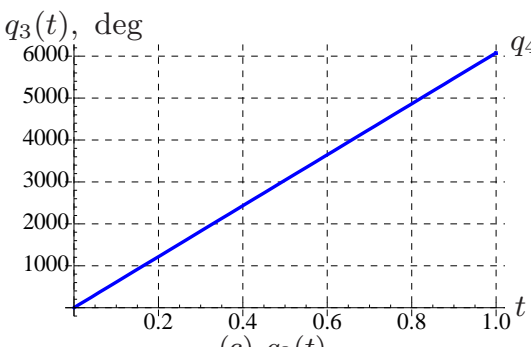

(c) $q_{3}(t)$

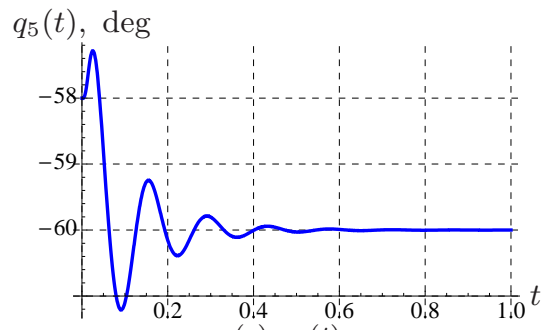

(e) $q_{5}(t)$

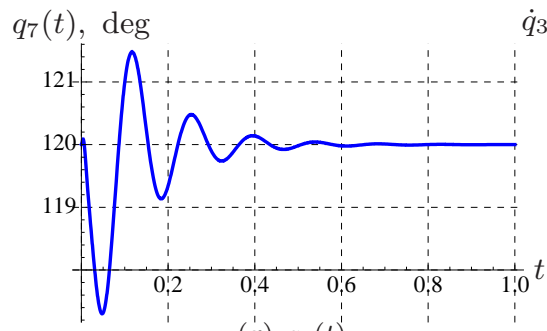

(g) $q_{7}(t)$

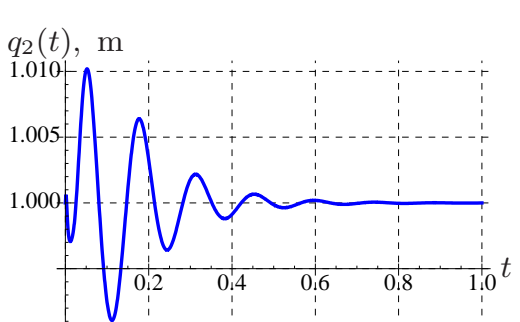

(b) $q_{2}(t)^{\prime}$

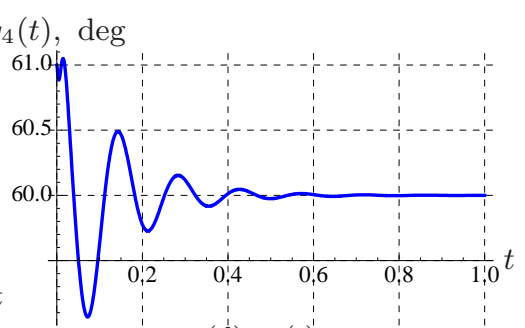

(d) $q_{4}(t)^{\prime}$

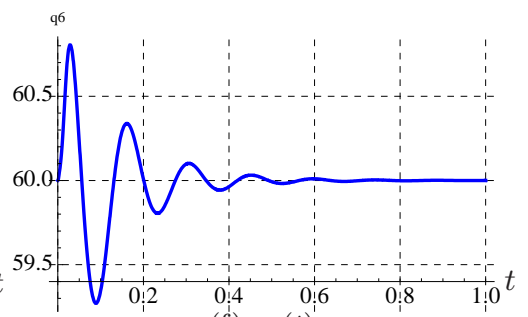

(f) $q_{6}(t)$

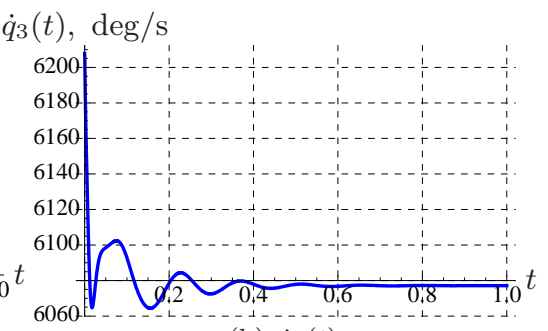

(h) $\dot{q}_{3}(t)$

Figure 7. Simulation results with linear feedback control to stabilize the third equilibrium.

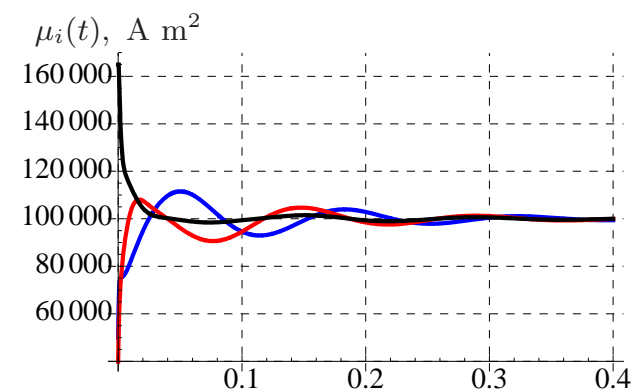

(a) $\mu_{1}(t)$ (blue), $\mu_{2}(t)$ (red), $\mu_{3}(t)$ (black)

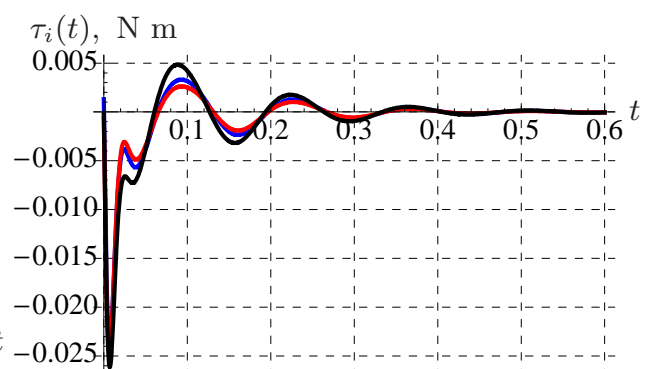

(b) $\tau_{1}(t)$ (blue), ${ }^{\prime} \tau_{2}(t)$ (red), $\tau_{3}(t)$ (black)

Figure 8. Control dipole moments and control torques with linear feedback control to stabilize the third equilibrium.

and showed that the system is controllable if one uses the magnetic dipole moments and control torques as 
inputs. The system was then stabilized using classical linearization-based feedback methods. Future work will focus on obtaining general stability and controllability conditions for the relative equilibria as well as the derivation of nonlinear control laws that outperform linearization-based control laws.

\section{ACKNOWLEDGEMENTS}

The research of Islam Hussein was supported by a WPI Faculty Development Grant. The research of Anthony Bloch was supported by NSF grants DMS-604307, and CMS-0408542.

\section{References}

${ }^{1}$ Hussein, I. I. and Schaub, H., "Invariant Shape Solutions of the Spinning Three Craft Coulomb Tether Problem," 2006 AAS/AIAA Space Flight Mechanics Meeting, 2006.

${ }^{2}$ Hussein, I. I. and Schaub, H., "Invariant Shape Solutions of the Spinning Three Craft Coulomb Tether Problem," Celestial Mechanics and Dynamical Astronomy, Vol. 96, 2006, pp. 137-157.

${ }^{3}$ Schaub, H. and Hussein, I. I., "Stability and Reconfiguration Analysis of a Circularly Spinning 2-Craft Coulomb Tether," Aerospace Science and Technology, 2007, Under review.

${ }^{4}$ Schaub, H. and Hussein, I. I., "Stability and Reconfiguration Analysis of a Circularly Spinning 2-Craft Coulomb Tether," 2007 IEEE Aerospace Conference, Big Sky, MT, 32007.

${ }^{5}$ Hussein, I. I. and Schaub, H., "Stability and Control of Relative Equilibria for the Three-Spacecraft Coulomb Tether Problem," AAS/AIAA Astrodynamics Specialists Conference, Mackinac Island, MI, Aug. 19-23 2007, Paper AAS 07-269.

${ }^{6}$ Elias, L. M., Dynamics of Multi-Body Space Interferometers Including Reaction Wheel Gyroscopic Stiffening Effects: Structurally Connected and Electromagnetic Formation Flying Architectures, Ph.D. thesis, Massachusetts Institute of Technology, 2004.

${ }^{7}$ Schweighart, S. A., Electromagnetic Formation Flight Dipole Solution Planning, Ph.D. thesis, Massachusetts Institute of Technology, 2005.

${ }^{8}$ Hussein, I. and Bloch, A., "Stability and Control of a Two-Craft Magnetic Constellation," AAS Spaceflight Mechanics Meeting, Galveston, TX, Jan. 27-31 2008, Paper No. AAS 08-189.

${ }^{9}$ Marsden, J. E., Lectures on Mechanics, Cambridge University Press, Cambridge, 1992.

${ }^{10}$ Laub, A. J. and Arnold, W. F., "Controllability and Observability Criteria for Multivariable Linear Second-Order Models," IEEE Transactions on Automatic Control, Vol. AC-29, No. 2, February 1984, pp. 163-165.

${ }^{11}$ Sanyal, A. K., Shen, J., and McClamroch, N. H., "Control of a Dumbbell Spacecraft using Attitude and Shape Control Inputs Only," Proceedings of the American Control Conference, Boston, MA, 2004, pp. 1014-1018. 